\title{
Monte Carlo analysis of neutron diffuse scattering data
}

\author{
D.J. Goossens ${ }^{\mathrm{a}, *}$, A.P. Heerdegen ${ }^{\mathrm{a}}$, T.R. Welberry ${ }^{\mathrm{a}}$, M.J. Gutmann ${ }^{\mathrm{b}}$ \\ ${ }^{a}$ Research School of Chemistry, The Australian National University, Canberra 0200, Australia \\ ${ }^{\mathrm{b}}$ Rutherford Appleton Laboratory, ISIS Facility, Chilton Didcot, Oxfordshire OX11 0QX, UK
}

\begin{abstract}
This paper presents a discussion of a technique developed for the analysis of neutron diffuse scattering data. The technique involves processing the data into reciprocal space sections and modelling the diffuse scattering in these sections. A Monte Carlo modelling approach is used in which the crystal energy is a function of interatomic distances between molecules and torsional rotations within molecules. The parameters of the model are the spring constants governing the interactions, as they determine the correlations which evolve when the model crystal structure is relaxed at finite temperature. When the model crystal has reached equilibrium its diffraction pattern is calculated and a $\chi^{2}$ goodness-of-fit test between observed and calculated data slices is performed. This allows a least-squares refinement of the fit parameters and so automated refinement can proceed. The first application of this methodology to neutron, rather than X-ray, data is outlined. The sample studied was deuterated benzil, d-benzil, $\mathrm{C}_{14} \mathrm{D}_{10} \mathrm{O}_{2}$, for which data was collected using time-offlight Laue diffraction on SXD at ISIS.
\end{abstract}

(C) 2006 Elsevier B.V. All rights reserved.

PACS: $61.12 . \mathrm{Ld} ; 61.10 . \mathrm{Nz} ; 61.66 . \mathrm{Hq}$

Keywords: Diffuse scattering; Automated refinement; Neutron scattering

\section{Introduction}

In a great many materials the disorder in the structure, static or dynamic, is crucial in determining the properties of the sample. Examining a small region of reciprocal space can give insight into particular modulations in the structure but only by modelling wide regions of reciprocal space can a detailed three-dimensional (3-d) picture of the disordered structure be assembled [1].

Here an approach to modelling large sets of diffuse scattering data [2] is extended to neutron scattering. In the past, the approach's quantitativeness has been compromised by data with a limited extent in reciprocal space. The magnitudes of atomic displacements are poorly defined if the data extends out to only small values of $\sin (\theta) / \lambda$. This became apparent in the study of the molecular crystal benzil, $\mathrm{C}_{14} \mathrm{H}_{10} \mathrm{O}_{2}$ (Fig. 1). A good fit was obtained to X-ray diffuse scattering collected in the laboratory [2] but when the same model was applied to neutron diffuse scattering

\footnotetext{
*Corresponding author. Tel.: +61261253536; fax: + 61261250750 .

E-mail address: goossens@rsc.anu.edu.au (D.J. Goossens).
}

data the magnitudes of the displacements were found to be too small by a factor of $\approx 2.5$, although the correlation structure determined from the X-ray study remained broadly applicable [3].

Neutron diffuse scattering is limited by source intensity as the diffuse peaks are generally $\sim 10^{-3}$ as bright as Bragg peaks. However, neutron diffraction at a pulsed source reaches far out in reciprocal space - useful data is obtained over three times farther out than for laboratory X-rays [3]. It is also capable of direct observation of deuterium (D) atoms, which is valuable because they are at the extremities of the molecule and undergo the greatest motions, giving a strong signal in the scattering. The use of neutron diffuse scattering data to refine a structural model is the aim of this work; the next step is refinement using neutron and X-ray diffuse data simultaneously.

\section{Experimental}

The neutron data were collected at ISIS using SXD. The data were not those published in Ref. [3] but the collection 
procedure was the same. This instrument uses neutron time-of-flight (tof) and detector angle to determine a $h \mathrm{kl}$ for each scattered neutron, and collects a 3-d volume of data which can be cut up into slices. For low-energy neutrons, intensity transfer due to inelasticity of scattering occurs, destroying pattern symmetry. There is useful information in this transfer, but as yet the program for calculation of diffuse scattering patterns [4] does not allow for changes in the length of the neutron wave vector. These inelastic effects were removed by the exclusion of low-energy neutrons, with the cut-off neutron energy being increased until the symmetry of the patterns was restored. The patterns presented use neutrons of greater than $20 \mathrm{meV}$ energy. This results in the large circular region of 'missing' data in Fig. 2(a).

\section{Results and discussion}

The analysis followed that discussed previously $[1,2,5]$. The molecule was described by a $z$-matrix which was placed in the crystal by a set of external coordinates - the position of the origin atom (given by a 3 -vector) and the orientation of the first defined bond in the molecule (given by a quaternion). A molecule of (in this case) 26 atoms could be described by six external variables (one of the elements of the quaternion is dependent) and three internal variables - these latter allow rotations around the three single $\mathrm{C}-\mathrm{C}$ bonds within the molecule (Fig. 1). The molecules interacted via Hooke's law springs, and these spring constants, plus those on the torsional degrees of freedom, were the parameters in the fit. These interactions induced correlations in the displacements and orientations of neighbouring molecules, giving features in the calculated diffuse scattering. By iteratively adjusting the spring constants, the model was refined. The values of the spring constants can be indicators of important interactions in the crystal but cannot be heavily interpreted as they are effective interactions only. Using atom-atom potentials for every pair of atoms in the crystal is computationally prohibitive.

The initial model (model I) was based on the second model proposed and described in detail in Ref. [2]. It used 11 spring constants between molecules $\left(F_{1}\right.$ to $\left.F_{11}\right)$ and one within $\left(F_{12}\right.$, referred to here as $\left.G_{1}\right)$ which applied to the

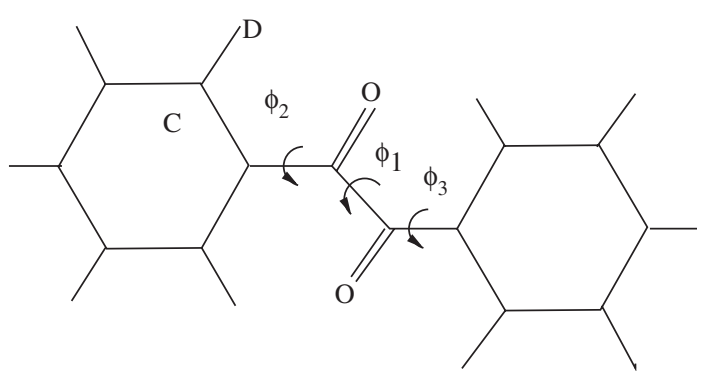

Fig. 1. Schematic diagram of the benzil molecule. The $\phi_{i}$ are the internal degrees of freedom.
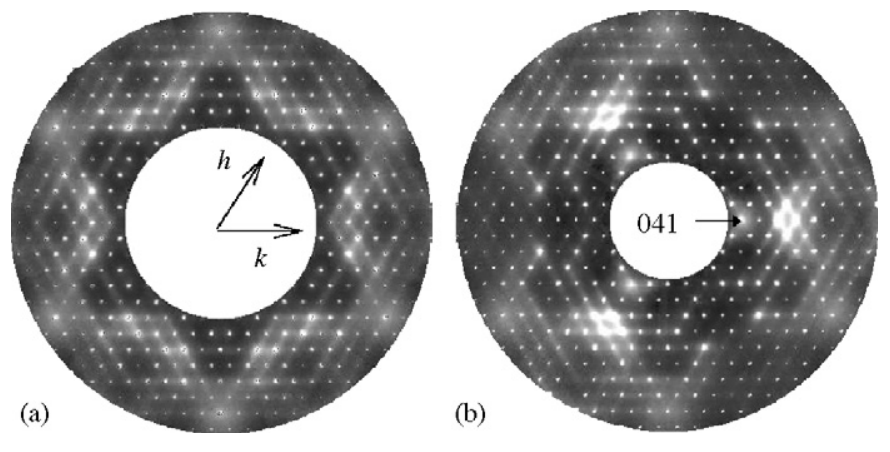

Fig. 2. Examples of observed data. (a) $=h k 0$ and (b) $=h k 1$.
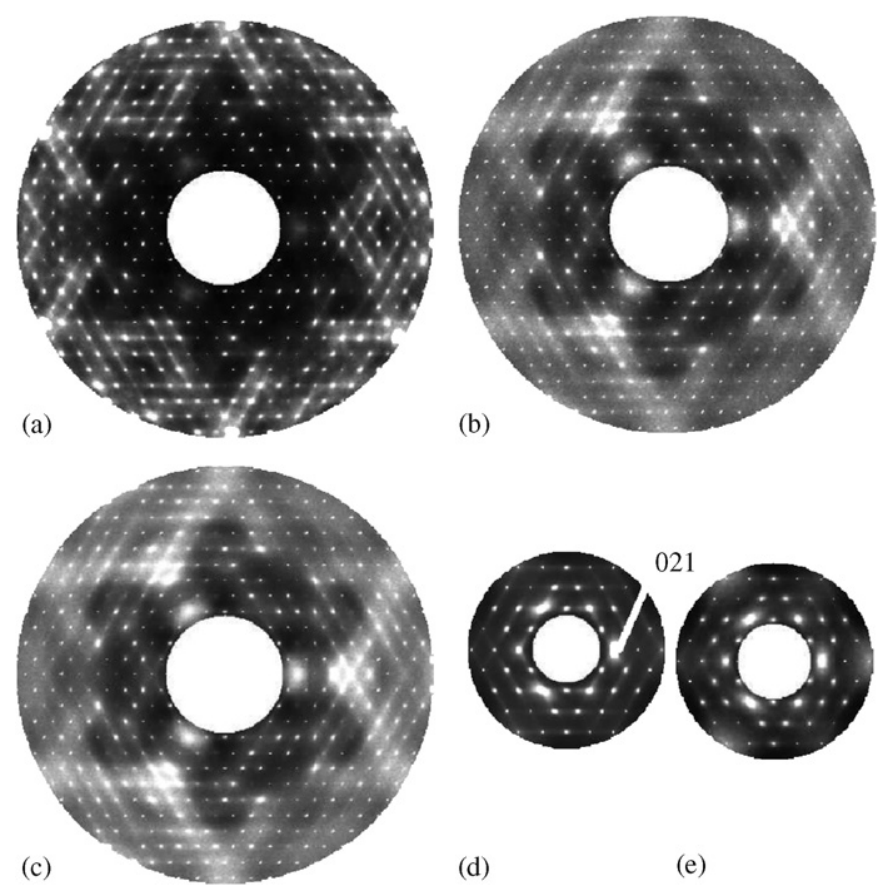

(b)

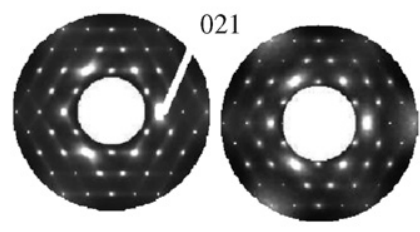

(d)

(e)

Fig. 3. (a) Calculated diffuse scattering in the $h k 1$ plane with spring constants from the X-ray fit [2]; sharp spots extend too far out. (b) Same as (a) but the spring constants have been globally scaled down by a factor of 30 (model I). (c) Calculated $h k 1$ plane when all springs were fitted independently (model II) (compare with Fig. 2b). (d) Observed X-ray diffuse scattering for $h k 1$, (e) X-ray diffuse scattering calculated from model II.

central bridge and the phenyl ring librations. Initially, the model was refined by scaling the springs globally; this was used to adjust the magnitudes of the displacements and correct the single largest flaw in the X-ray model. The Xray model used an 'overall' $B$-factor, $B_{\text {overall }}$, to 'soak up' much of the isotropic disorder in the crystal. This factor is not used here.

Planes $h k 0, h k 1$ and $h k 2$ were used in the fitting but for space reasons Fig. 3 shows calculated scattering for $h k 1$ only. For model I the factor for scaling down the spring constants relative to the X-ray model was found to be $30 \pm$ 5 (Fig. 3b). In model II, the spring constants were allowed to vary independently during the fitting. Model II was also applied to X-ray data to test consistency (Figs. 3d and e). 
Table 1

Comparison of isotropic $B$ factors from single crystal refinement (calculated from results in Ref. [6]) with those from model II

\begin{tabular}{llllll}
\hline $\begin{array}{l}\text { Atom label } \\
\text { (see Ref. [6]) }\end{array}$ & $\begin{array}{l}B_{\text {iso }} \\
{[6]}\end{array}$ & $\begin{array}{l}B_{\text {iso }} \AA^{2} \\
(\text { model II) }\end{array}$ & Atom & $\begin{array}{l}B_{\text {iso }} \\
{[6]}\end{array}$ & $\begin{array}{l}B_{\text {iso }} \AA^{2} \\
\text { (model II) }\end{array}$ \\
\hline C0 & 3.32 & 3.17 & C3 & 6.16 & 4.77 \\
O & 4.74 & 4.94 & C4 & 6.32 & 4.80 \\
C1 & 3.00 & 2.82 & C5 & 4.97 & 4.85 \\
C2 & 3.86 & 3.86 & C6 & 3.39 & 3.77 \\
\hline
\end{tabular}

Table 2

Comparison of spring constants for models I (see Ref. [2] for a detailed description of these springs) and II

\begin{tabular}{llllll}
\hline $\begin{array}{l}\text { Spring } \\
\text { type }\end{array}$ & Model I & Model II & $\begin{array}{l}\text { Spring } \\
\text { type }\end{array}$ & Model I & Model II \\
\hline$F_{1}$ & 4.0 & $4.2(1)$ & $F_{7}$ & 4.9 & $6.2(2)$ \\
$F_{2}$ & 1.6 & $2.9(1)$ & $F_{8}$ & 2.6 & $4.8(2)$ \\
$F_{3}$ & 0.5 & $1.3(1)$ & $F_{9}$ & 4.0 & $6.9(2)$ \\
$F_{4}$ & 2.9 & $3.0(1)$ & $F_{10}$ & 2.7 & $5.4(2)$ \\
$F_{5}$ & 1.2 & $1.3(1)$ & $F_{11}$ & 1.3 & $1.8(1)$ \\
$F_{6}$ & 0.6 & $1.8(1)$ & $G_{1}$ & 0.0053 & $0.0015(1)$ \\
\hline
\end{tabular}

Values are relative to $k_{\mathrm{B}} T=1$.

Table 1 compares isotropic thermals factors, $B_{\text {iso }} \mathrm{s}$, from model II with those derived from the literature study of the average structure of hydrogenous benzil [6] (for heavy atoms as the literature study used X-rays). As expected given that the atoms our modelling are constrained using rigid bodies the values are not identical, but are in all cases quite similar.

Table 2 compares spring constants from the two neutron models (note: those for model I are the X-ray spring constants from Ref. [2] scaled down by a factor of 30). Large constants have remained large and small have remained small. In general those for model II are larger than for model I. $G_{1}$ has fallen considerably, which is reasonable; there is no $B_{\text {overall }}$ to artificially account for molecular motions, so the molecule becomes less rigid to allow the atomic displacements to grow.

The largest absolute increases are in $F_{8}, F_{9}$ and $F_{10}$. These springs all propagate along the $c$-axis [2], and their strengthening indicates that the large $Q$ range of the neutron data better constrains the correlations perpendi- cular to the planes of the data. A next step is to use planes orthogonal to those presented here to improve this further.

Initial modifications of model II show that the internal spring constants, which could be treated as identical in the X-ray fitting, become well differentiated, due to the sensitivity of the neutron to the $\mathrm{D}$ atoms and thus to the librational motions of the molecules.

\section{Conclusions}

The refinement techniques used for X-ray diffuse scattering $[1,2]$ could be extended to neutron diffraction data if it was ensured that effects on the intensity distribution due to inelastic scattering were eliminated. The large $Q$ range of single crystal data collected on a tof instrument allowed a more quantitative modelling of the atomic displacement parameters, while the sensitivity of neutrons to deuterium allowed the torsional spring constants to be better defined as the $\mathrm{D}(\mathrm{H})$ atoms lie at the extremities of the molecule and undergo the greatest motions. In future, extraction of multiple reciprocal space sections in different orientations will allow further tests of the 3-d modelling of the diffuse scattering, and finally the combination of such refinements with X-ray diffraction data, whether laboratory or synchrotron, will allow the short range structure in many materials to be elucidated at a level of detail previously unattainable.

\section{Acknowledgements}

The authors thank the Access to Major Research Facilities Program and the Australia Research Council for financial support, the Australian Partnership for Advanced Computing for computer time and Prof. W.I.F. David (ISIS) for help with sample procurement.

\section{References}

[1] T.R. Welberry, Diffuse X-ray Scattering and Models of Disorder, OUP, 2004.

[2] T.R. Welberry, D.J. Goossens, A.J. Edwards, W.I.F. David, Acta Cryst. A 57 (2001) 101.

[3] T.R. Welberry, D.J. Goossens, W.I.F. David, M.J. Gutmann, M.J. Bull, A.P. Heerdegen, J. Appl. Cryst. 36 (2003) 1440.

[4] B.D. Butler, T.R. Welberry, J. Appl. Cryst. 25 (1992) 391.

[5] D.J. Goossens, T.R. Welberry, A.P. Heerdegen, Z. Krist. 220 (2005) 1035.

[6] M. More, G. Odou, J. Lefebvre, Acta Crystallogr. B 43 (1987) 398. 